\title{
Complements to the longitudinal librations of an elastic 3-layer Titan on a non-Keplerian orbit
}

\author{
Andy Richard ${ }^{1,2}$ and Nicolas Rambaux ${ }^{1,2}$ \\ ${ }^{1}$ IMCCE, Observatoire de Paris, UMR 8028, CNRS, UPMC \\ 77 avenue Denfert-Rochereau, 75014 Paris, France \\ email: arichard@imcce.fr \\ ${ }^{2}$ Université Pierre et Marie Curie, UPMC - Paris 06, \\ 4 Place Jussieu, 75005 Paris \\ email: Nicolas.Rambaux@imcce.fr
}

\begin{abstract}
Titan longitudinal librations are dependent on the satellite internal structure and the elastic behavior of the surface. The elastic deformation of the surface is related to the perturbing potential through the Love theory. In a previous paper, we described the deformation as a response to the tidal potential exerted by Saturn at orbital frequency. Here we improve the tidal deformation reponse by including the effect of the libration angle and the orbital perturbations. We then provide the libration amplitudes associated with the rotational model of a tidally deformed three-layer Titan evolving on a non-Keplerian orbit.
\end{abstract}

Keywords. planets and satellites: individual (Titan), librations, rotation, elasticity, perturbations

\section{Introduction}

Goldreich and Mitchell (2010) suggested that the librational motion of icy satellites should be strongly reduced when the icy shell behaves elastically. In the case of an elastic icy shell, the surface should deform instead of rotate under the influence of the gravitational potential of the planet. In the case of Titan, the reduction of the longitudinal libration has been assessed by Van Hoolst et al. (2013) and Jara-Orué and Vermeersen (2014) at the orbital frequency and Richard et al. (2014) for elastic three-layered Titan evolving on a non-Keplerian orbit. Richard et al. (2014) computed the deformations of the surfaces by using the Love theory and the perturbing tidal potential detailed by Giampieri (2004). We analyzed the Horizons ephemerides (Giorgini et al. 1996) of Titan to obtain the frequencies of the perturbed orbit present in the gravitational torque exerted by Saturn. By taking into account the elasticity, two new libration frequencies were identified. Here we complete the results obtained in the paper of Richard et al. (2014) by including the libration angle and the orbital perturbations in the deformation process and evaluating the libration amplitude resulting at different orbital forcing frequencies in the case of a non-Keplerian orbit.

\section{Rotational model}

The rotational dynamics of Titan composed of an icy shell, an internal ocean and a solid inner core can be given by the Euler equations written for each layer in the Tisserand reference frame of the satellite by taking into account the external and internal gravitational torques and the ocean pressure torque (see e.g. Richard et al. 2014). 
Under the tidal potential of Saturn, the surfaces of the layers composing Titan are deformed. Thus, the mass distribution depends on the time and the inertia tensor can be decomposed into a static and a dynamic part such as $\mathcal{I}=\mathcal{I}_{0}+\Delta \mathcal{I}(t)$. The dynamic part of the inertia tensor can be obtained by using the Love theory describing the deformation under the influence of a perturbing potential.

The perturbing tidal potential $W$ for a synchronous satellite is expressed by Giampieri (2004) when the libration angle is neglected. As a complement to the paper of Richard et al. (2014), the libration angle and the orbital perturbations are included in the potential which is then written in spherical coordinates $\left(r_{o}, \theta, \phi\right)$ with $r_{o}$ the mean radius of the layer considered, $\theta$ and $\phi$ the colatitude and longitude respectively

$$
\begin{aligned}
W\left(r_{o}, \theta, \phi\right)=-\frac{G M}{a^{3}} r_{o}^{2}\left(\frac{a}{r}\right)^{3}\left[-\frac{1}{2} P_{2}^{0}(\cos \theta)\right. & +\frac{1}{4} P_{2}^{2}(\cos \theta) \cos 2 \phi \cos 2(\nu-L-\gamma) \\
+ & \left.\frac{1}{4} P_{2}^{2}(\cos \theta) \sin 2 \phi \sin 2(\nu-L-\gamma)\right]
\end{aligned}
$$

where $G$ is the gravitational constant, $M$ is the mass of the planet, $a$ is the semi-major axis of Titan orbit, $P_{2}^{j}$ are the associated Legendre polynomials of degree $2, \nu$ is the true longitude, $L$ is the mean longitude and $\gamma$ is the libration angle.

The gravitational torque exerted by Saturn on Titan surface depends on the position of the planet expressed in the reference frame of the satellite. We use the rotation matrices $R_{1}$ and $R_{3}$ to define cartesian coordinates $(x, y, z)$ of the planet expressed in Titan frame such as $(x, y, z)^{T}=R_{3}(\varphi) R_{1}(\Theta) R_{3}(\Psi) R_{3}(-\Omega) R_{1}(-i) R_{3}(-\omega)\left(r \cos f_{p}, r \sin f_{p}, 0\right)^{T}$ where $f_{p}$ is the true anomaly of the planet, $\omega, i$ and $\Omega$ are the classical orbital elements, and $\varphi, \Theta$ and $\Psi$ are the Euler angles. By using the Saturn equatorial plane as the inertial reference frame, $\Theta$ and $i$ can be neglected.

The longitude of the planet in the reference frame of the satellite is given by $\phi_{p}=$ $\Omega+\omega+f_{p}-\Psi-\varphi$. We use the definition of Eckhardt (1965) $\Psi+\varphi=\pi+L_{s}+\gamma$, where $L_{s}$ is the mean longitude of the satellite defined as $L=N t+L_{0}$ with $N$ being the mean mean motion (Vienne and Duriez 1995) and $L_{0}$ its phase. Thus, the libration angle $\gamma$ includes the periodic variations of the orbital motion. By using the true longitude of the satellite defined as $\nu=\Omega+\omega+f=\pi+\nu_{p}, \nu_{p}$ being the true longitude of the planet expressed in the satellite reference frame, the set of planet coordinates can be written as functions of $\nu-L$ and $\gamma$.

By including the libration angle in the deformations, the external and internal gravitational torques exerted on the icy shell provided by the expressions (27) and (29) of Richard et al. (2014) can be generalized as

$$
\begin{aligned}
\Gamma_{e x t}= & \frac{1}{2}\left(\frac{a}{r}\right)^{3}\left(K_{s}-\frac{K_{s}^{p}}{3}+K_{i n t}^{s / p}-\frac{K_{i n t}^{p / p}}{8}\right) \sin 2\left(\nu-L-\gamma_{s}\right), \\
\Gamma_{i n t}= & \left(K_{i n t}-\frac{K_{i n t}^{s / p}}{2}-\frac{K_{i n t}^{p / s}}{12}+\frac{K_{i n t}^{p / p}}{16}\right) \sin 2\left(\gamma_{i}-\gamma_{s}\right) \\
& -\left(\frac{a}{r}\right)^{3}\left(\frac{K_{i n t}^{p / s}}{12}-\frac{K_{i n t}^{p / p}}{16}\right) \sin 2\left(\nu-L-\gamma_{i}\right),
\end{aligned}
$$

with $\gamma_{s}$ and $\gamma_{i}$ the libration angles of the icy shell and the inner core, $K_{s}$ and $K_{s}^{p}$ the amplitudes of the torques exerted on the static and dynamic figures of the shell respectively, $K_{\text {int }}$ and $K_{\text {int }}^{s / p}$ the amplitudes of the internal gravitational torques exerted by the static figure of the shell on the static and dynamic figure of the inner core respectively, $K_{i n t}^{p / s}$ and $K_{i n t}^{p / p}$ the amplitudes of the internal torques exerted by the dynamic figure of the shell on the static and dynamic figure of the inner core respectively. All these amplitudes 
Table 1. Frequency analysis of $\nu-L$ for Titan obtained with the JPL Horizons ephemerides taken over 400 years. The identification is made with the TASS ephemerides. The magnitudes are truncated at $30 "$ ". The initial date is J2000.

\begin{tabular}{rlrccr}
\hline $\boldsymbol{i}$ & $\begin{array}{l}\text { Frequency } \\
\text { (rad/days) }\end{array}$ & $\begin{array}{r}\text { Period } \\
\text { (days) }\end{array}$ & $\begin{array}{c}\text { Magnitude } \\
\text { (") }\end{array}$ & $\begin{array}{c}\text { Phase } \\
\text { (degrees) }\end{array}$ & Identification \\
\hline 1 & 0.394018 & 15.9464 & 11899.3237 & 163.3693 & $L_{6}-\phi_{6}$ \\
2 & 0.788036 & 7.9732 & 212.5868 & -32.7941 & $2 L_{6}-2 \phi_{6}$ \\
3 & 0.394081 & 15.9439 & 56.6941 & -68.1211 & $L_{6}-\Phi_{6}$ \\
4 & 0.001169 & 5376.6331 & 43.7313 & -66.0428 & $2 L_{9}$ \\
5 & 0.000584 & 10750.3648 & 37.5508 & 138.4821 & $L_{9}$ \\
6 & 0.392897 & 15.9919 & 31.5673 & 10.8789 & $L_{6}-\Phi_{6}-2 L_{9}$ \\
\hline
\end{tabular}

are defined in Richard et al. (2014). The presence of the libration angle in the torques will induce variations of the proper frequencies, especially due to the elastic term $K_{s}^{p}$ which is of the same order of magnitude as the rigid torque amplitude $K_{s}$.

\section{Orbital motion}

The Saturn gravitational torque exerted on the multi-layered satellite depends on the orbital motion of the satellite expressed through $\nu-L$ in $(2.2)$. Due to interaction with the Sun, the planets and other satellites, the orbital motion of Titan is perturbed and $\nu-L$ can be approximated by a Fourier series such as (Vienne and Duriez 1995) $\nu-$ $L=\sum_{j=0}^{\infty} H_{j} \sin \left(\omega_{j} t+\alpha_{j}\right)$, where $\omega_{j}$ are the frequencies of the orbital motion, $H_{j}$ the magnitudes and $\alpha_{j}$ the phases.

In Table 1, the frequency analysis of $\nu-L$ obtained with the Horizons ephemerides (Giorgini et al. 1996) provided over 400 years is detailed. We used the notation of Vienne and Duriez (1995) where $\phi$ is close to the longitude of the periapsis, $\Phi$ is close to the longitude of the node and subscripts 6 and 9 refer to Titan and the Sun respectively. In the paper of Richard et al. (2014), the short period of the ephemerides compared to the evolution rate of the longitude of the node and the periapsis argument ( $\sim 703$ years) lead us to an incorrect identification of a frequency that we attributed to a perturbation of Iapetus. The comparison with frequency analysis of the TASS ephemerides of Vienne and Duriez (1995) taken over 30000 years allowed to identify a better candidate.

Indeed, the determination of the third term of the frequency analysis (Table 1) is problematic due to the proximity of its frequency with the first term. With Horizons, the frequency is determined at $0.394081 \mathrm{rad} /$ days and has a difference of $\pm 0.000014 \mathrm{rad} /$ days with the frequency determined with TASS. This difference is due to the short time scale of 400 years of ephemerides which is insufficient to precisely determine the third term frequency. However, TASS permitted to identify the argument of this term as $L_{6}-\Phi_{6}$.

\section{Librational motion}

The librational motion in longitude of Titan is decomposed at the different orbital forcing frequencies. The variations of the inertia tensor due to the perturbed orbital motion and the librations are included in the libration equations for the ice shell and the solid inner core. The solutions are then obtained by decomposing the system over each perturbing frequency.

The amplitudes of the system are obtained analytically and listed in Table 2. Here we used the interior model CA10 described in Richard et al. (2014) and derived from Castillo-Rogez and Lunine (2010). The presence of terms coming from the torques exerted 
Table 2. Analytical libration amplitudes of the icy shell (expressed as equator deviation in meters) for the CA10 interior model of Titan at different forcing frequencies. The last column describes the difference of amplitudes between the librational models.

\begin{tabular}{llrrr}
\hline $\begin{array}{l}\text { Freq. } \\
\text { (rad/days) }\end{array}$ & $\begin{array}{c}\text { Period } \\
\text { (days) }\end{array}$ & (Richard et al. $(2014))$ & $\begin{array}{c}\text { CA10 } \\
\text { (this paper) }\end{array}$ & $\begin{array}{c}\text { Amp. diff. } \\
\text { (\%) }\end{array}$ \\
\hline 0.394018 & 15.9464 & $-72.751 \mathrm{~m}$ & $-73.142 \mathrm{~m}$ & 0.54 \\
0.001169 & 5376.63 & $552.265 \mathrm{~m}$ & $574.051 \mathrm{~m}$ & 3.94 \\
0.000584 & 10750.4 & $470.171 \mathrm{~m}$ & $474.673 \mathrm{~m}$ & 0.96 \\
0.000063 & $99,027.4$ & $51.963 \mathrm{~m}$ & $30.523 \mathrm{~m}$ & 41.26 \\
0.001121 & 5606.25 & $29.185 \mathrm{~m}$ & $17.792 \mathrm{~m}$ & 39.04 \\
\hline
\end{tabular}

on the dynamic figure of the different layers modifies the proper frequencies $\omega_{1}$ and $\omega_{2}$ of the system obtained in the rigid case by about $52 \%$ for $\omega_{1}$ and $2 \%$ for $\omega_{2}$ compared to the values of Richard et al. (2014). These variations of the proper frequencies are of the same order as those obtained in the model of libration at orbital frequency of Van Hoolst et al. (2013).

The libration amplitudes at orbital frequency are strongly reduced when compared to the rigid case where the amplitudes are higher by about a factor 5 . The librational angle included in the deformation process modifies the amplitudes at the orbital frequency by less than $1 \%$. However, by including the librations and orbital perturbations in the deformations, the responses at low frequencies are increased, as for the dominant saturnian annual term increased by about 15 meters, or about $4 \%$. These variations can be attributed to the modification of the proper frequencies due to the tidal perturbing potential and the deformation. Finally, the two frequencies of 0.000063 and $0.001121 \mathrm{rad} /$ days described by Richard et al. (2014) and resulting from the orbital forcing exerted on the dynamic figure of the satellite are less dependent on the internal ocean. Their amplitudes for the oceanic models are reduced by about $40 \%$ when the libration angle is included in the deformations and are now of the same order as for the solid model. The variations between models are below one meter.

The signature of the internal structure exists in the high frequency forced libration but its amplitude is strongly reduced when compared to the rigid case. The detection of such a signature is thus challenging.

\section{Acknowledgments}

The authors wish to thank Alain Vienne for the fruitful discussions on the frequency analysis of the TASS ephemerides. The authors are also thankful to the IAU and the CS of Paris Observatory for their financial support.

\section{References}

Castillo-Rogez, J. \& Lunine, J. I. 2010, Geophys. Res. Lett., 37, 20205

Eckhardt, D. H. 1965, AJ, 70, 466

Giampieri, G. 2004, Icarus, 167, 228

Giorgini, J. D., et al. 1996, AAS, 28, 1158

Goldreich, P. M. \& Mitchell, J. L. 2010, Icarus, 209, 631

Jara-Orué, H. M. \& Vermeersen, B. L. A. 2014, Icarus, 229, 31

Richard, A., Rambaux, N., \& Charnay, B. 2014, Planet. Space Sci. 93, 22

Van Hoolst, T., Baland, R.-M., \& Trinh, A. 2013, Icarus, 226, 299

Vienne, A. \& Duriez, L. 1995, A\&GA, 297, 588 